\title{
Karl Juhnke
}

Das Erzählmotiv des Serienmörders im Spielfilm 
DVV Sozialwissenschaft 


\section{Karl Juhnke}

\section{Das Erzählmotiv des Serienmörders im Spielfilm}

Eine filmwissenschaftliche Untersuchung

Mit einem Geleitwort von Prof. Dr. Georg Kiefer 
Die Deutsche Bibliothek - CIP-Einheitsaufnahme

Juhnke, Karl:

Das Erzählmotiv des Serienmörders im Spielfilm : eine

filmwissenschaftliche Untersuchung / Karl Juhnke.

Mit einem Geleitw. von Georg Kiefer.

- 1. Aufl.. - Wiesbaden : Dt. Univ.-Verl., 2001

(DUV: Sozialwissenschaft)

Zugl.: Braunschweig, Hochsch. für Bildende Künste, Diss., 2000

ISBN 978-3-8244-4454-0 ISBN 978-3-322-89955-2 (eBook)

DOI 10.1007/978-3-322-89955-2

1. Auflage Mai 2001

Alle Rechte vorbehalten

(C) Springer Fachmedien Wiesbaden 2001

Ursprünglich erschienen bei Deutscher Universitäts-Verlag GmbH, Wiesbaden, 2001.

Lektorat: Ute Wrasmann / Dr. Tatjana Rollnik-Manke

www.duv.de

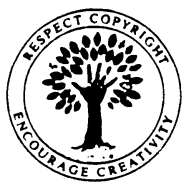

Das Werk einschließlich aller seiner Teile ist urheberrechtlich geschützt. Jede Verwertung außerhalb der engen Grenzen des Urheberrechtsgesetzes ist ohne Zustimmung des Verlags unzulässig und strafbar. Das gilf insbesondere für Vervielfältigungen, Übersetzungen, Mikroverfilmungen und die Einspeicherung und Verarbeitung in elektronischen Systemen.

Die Wiedergabe von Gebrauchsnamen, Handelsnamen, Warenbezeichnungen usw. in diesem Werk berechtigt auch ohne besondere Kennzeichnung nicht zu der Annahme, dass solche Namen im Sinne der Warenzeichen- und Markenschutz-Gesetzgebung als frei zu betrachten wären und daher von jedermann benutzt werden dürften.

Gedruckt auf säurefreiem und chlorfrei gebleichtem Papier. 


\section{Geleitwort}

Oder: Die aristotelische Gänsehaut führt nicht zur Katharsis

Filmwissenschaftler bin ich nicht. Kulturwissenschaftler schon eher. D.h. Liebhaber von guten Filmen bin ich schon, aber kein Filmhistoriker, der Genres und Motive exakt analysiert.

Die vorliegende Arbeit habe ich dennoch von Anfang an mit Interesse begleitet, da es zur detektivischen Arbeit gehört, beim Ermitteln die richtige Methode zu finden. Es handelt sich um eine massenmediale Untersuchung und „Was wir über unsere Gesellschaft, ja über die Welt, in der wir leben, wissen, wissen wir durch die Massenmedien“ (Luhmann).

Was wissen wir, wenn wir über Serienmörderfilme etwas wissen? Wissen wir dann etwas über die Serienmörder? Oder über die Konstruktion der Filme? Oder über die Perzipienten/Konsumenten, die nach dieser Ware verlangen? Warum hat diese Filmsparte einen solchen Boom in den USA und Westeuropa?

Während ich diesen Text schreibe, lese ich in der Zeitung:

„Schwerbewaffneter tötete Ex-Kollegen.

Melrose Park, 6.2. (ap) Einen Tag vor Beginn seiner Haftstrafe hat ein 66-jühriger, ehemaliger Angestellter einer Fahrzeugfabrik in Chicago vier Menschen und sich selbst erschossen. Vier weitere Mitarbeiter wurden zum Teil lebensgefährlich verletzt. William Baker hatte 39 Jahre lang in der Firma gearbeitet, war aber 1994 wegen Diebstahls entlassen worden. Er war dafür zu fünf Monaten Haft verurteilt worden. Zehn bis 15 Minuten lang zog Baker mit mehreren Schusswaffen in einer Golftasche durch das Werksgelände des LKW-Herstellers Navistar. Er schoss mit einem AK-47 Sturmgewehr um sich und traf mindestens acht Menschen.

Vor eineinhalb Monaten hatte ein Softwareprogrammierer der Firma Edgewater Technology im Staat Massachusetts sieben Menschen erschossen. Die Steuerhehörde wollte einen Teil seines Lohnes pfänden. Im März 2000 erschoss ein 29-Jähriger nach seiner Entlassung aus einer Autowaschanlage in Dallas fünf Menschen. Im November 1999 hatte ein Wartungsmechaniker für Kopierer in der Firma Xerox in Honolulu offenbar wegen Problemen am Arbeitsplatz sieben Kollegen erschossen.“

Nun, dies sind keine Serienmörder, sondern Amokläufer, und es sind auch keine Filme, von denen die Rede ist, sondern es ist Wirklichkeit. Und doch, hat dies mit dem zu tun, was wir von den Medien wissen? Oder hat es mit der Arbeit zu tun oder mit der „Ästhetik des Schreckens“? 
Wer die einschlägigen Statistiken kennt, weiß, dass die Zahl der Morde die Zahl der Filme um ein Vielfaches übersteigt.

Robert Redford, der ja ein engagierter Umweltschützer ist, äußert in einem Interview vom selben Tag:

„Das Thema (Umweltschutz), selbst wenn es in den Medien ist, wird nicht mehr wahrgenommen, weil es kein Charisma, keinen Sex-Appeal hat.“

Frage: Und nicht mal Robert Redford hat die Öko-Themen sexy präsentieren können?

„Wissen Sie, in den USA sind heute Serien-Mörder sexy. Der Umweltschutz ist es nicht.

(...) Die Themen müssen gewalttätig und extrem sein, um überhaupt noch Aufmerksamkeit zu erregen. Denn in der Mediengesellschaft von heute lässt unsere Aufmerksamkeit ständig nach." (Frankfurter Rundschau vom 03.02.2001)

Wieder geht es um den Zusammenhang von Medien und Gewalt, wobei die Wirklichkeit die „Wirklichkeit der Medien“ anstachelt, sex\&crime die höchste Aufmerksamkeit erlangen, als Fiktion. Es handelt sich ja nicht um Kriegsfilme mit legalisiertem Mord, nicht um das Aufstürmen der Nationalgarden usf.

Handelt es sich um die Freiheit, selbst zu richten, um die Freiheit, selber Ordnung zu schaffen? Warum boomt diese Filmsparte? Weil sie sich rechnet. Warum rechnet sie sich?

Der Autor hat knapp 600 Serienmörderfilme erduldet. Er gibt einen Abriss zur Geschichte des Themas, er setzt unterschiedliche Methoden der Mengenuntersuchung ein, bietet exemplarische Analysen an und - als Ermittler - entwickelt er Erklärungsmuster.

Was er dabei herausfindet, soll hier allerdings nicht verraten werden.

Prof. Dr. Georg Kiefer 


\section{Vorwort}

Serienmörder erfreuen sich in den letzten zwei Jahrzehnten einer breiten Aufmerksamkeit in Spielfilmen und anderen narrativen Kontexten. Ihr massenhaftes Auftreten als Film-(anti-)helden bildete den Ausgangspunkt meiner Auseinandersetzung: Was macht die Serienmörder so ungemein attraktiv für Filmproduzenten wie -rezipienten?

Um eine verlässliche Basis für diese Untersuchung zu erhalten, wurde nicht allein der exemplarischen Filmanalyse vertraut, sondern eine Massenuntersuchung der zugänglichen Serienmörderfilme durchgeführt. Weiterhin erwies es sich als sinnvoll, einen thematischen Zugriff zu wählen, da er im Gegensatz zum in der Filmwissenschaft gebräuchlicheren Genreansatz Narrationsinhalte wesentlich trennschärfer greifbar macht. Serienmörder kommen in Horrorfilmen, Thrillern, Krimis oder Komödien vor, und das in unterschiedlichen Graden der Handlungsrelevanz. Für die Analyse bot sich daher der aus der Literaturwissenschaft bekannte Motivbegriff an.

Ziel dieser Motivanalyse ist es, Ausmaß, Tradition, Funktion und Funktionalisierung des Serienmörders im Spielfilm zu erschließen. Dabei geht es um die grundlegenden gesellschaftlichen Diskurse von Gewalt, Sexualität und Gender in verschiedenen Formen ihrer filmischen Ästhetisierung.

Die vorliegende Arbeit wurde 1999 an der Hochschule der Bildenden Künste in Braunschweig zur Promotion angenommen.

Danken möchte ich an dieser Stelle allen, die zum Gelingen dieser Arbeit beigetragen haben: den Gutachtern Prof. Dr. Helmut Korte und Prof. Dr. Georg Kiefer; vielen Gesprächspartnern, die Anregungen und Kritik lieferten, und besonders Regina Nußbaum für kontinuierliche und vielfältige Unterstützung.

Karl Juhnke 


\section{Inhaltsverzeichnis}

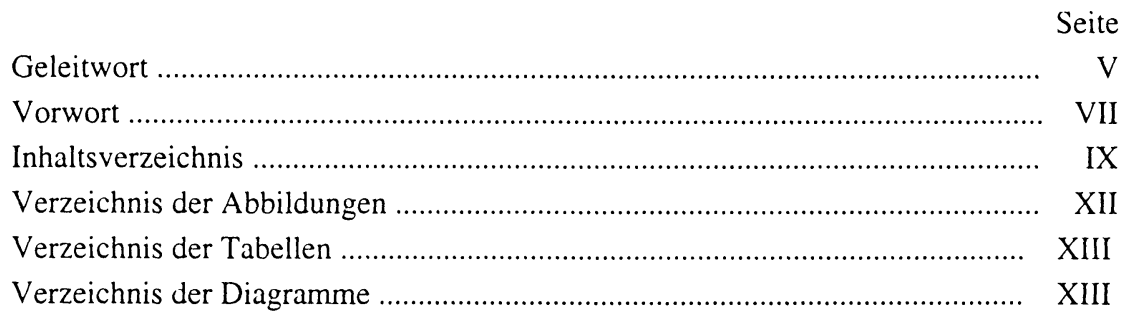

1. Das Erzählmotiv des Serienmörders im Spielfilm ........................................... 1

2. Grundlagen der Untersuchung .................................................................... 5

2.1. Was sind Serienmörder? ................................................................ 5

2.2. Motivforschung …………………………………………………..... 7

2.2.1. Beispiel Western .............................................................. 12

2.2.2. Beispiel Horrorfilm.............................................................. 14

2.2.3. Beispiel Vampyrfilm ........................................................... 16

2.2.4. Das Serienmördermotiv ...................................................... 17

2.3. Untersuchungssample...................................................................... 19

2.4. Zum Hintergrund filmwissenschaftlicher Untersuchungen .................. 21

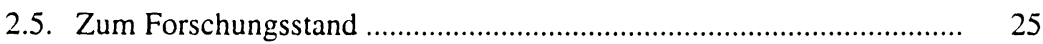

2.6. Methodischer Ablauf einer filmwissenschaftlichen
Motivuntersuchung ............................................................................ 26

3. Umfang der Serienmörderfilmproduktion ........................................................ 31

3.1. Historische Entwicklung des Serienmördermotivs im Spielfilm .......... 31

3.2. Quantitative Entwicklung des Serienmördermotivs in Spielfilmproduktionen ................................................................... 37

3.2.1. Serienmörderfilme und Produktionsländer .............................. 43

3.2.2. Genrezugehörigkeit ............................................................ 45

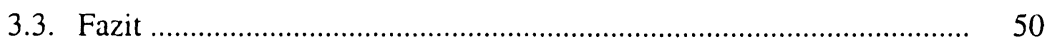

4. Quantitative Bestimmung des Serienmördermotivs im Spielfilm ...................... 53

4.1. Die Triade Täter - Opfer - Ermittler ……………………………….... 53

4.2. Serienmörder im Mittelpunkt ............................................................. 58

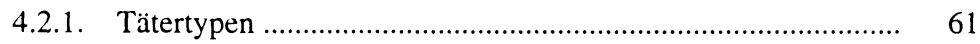

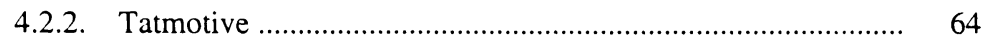

4.2.3. Weitere Tätercharakteristika .................................................... 67 


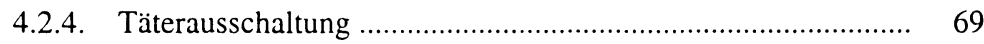

4.3. Opfer im Mittelpunkt ....................................................................... 70

4.4. Ermittler im Mittelpunkt ................................................................... 76

4.5. Rezeptionsangebote .................................................................. 82

4.5.1. Gewalt ....................................................................... 84

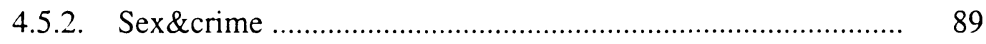

4.5.3. Slasher .................................................................... 92

4.5.4. Formale Spannungselemente............................................. 93

4.5.5. Versatzstücke des Serienmörderfilms .................................... 96

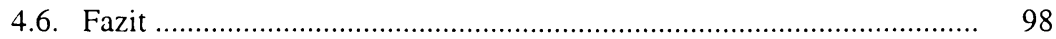

5. Das Serienmördermotiv und seine Funktion im Spielfilm ........................... 103

5.1. Der Gebrauch des Serienmördermotivs im Spielfilm ..................... 103

5.2. Exemplarische Filmanalysen ....................................................... 112

5.2.1. Die Filmauswahl ......................................................... 112

5.2.2. Zur Filmanalyse .............................................................. 114

5.3. BIZARRE MORDE ........................................................................ 115

5.3.1. Sequenzprotokoll ......................................................... 116

5.3.2. Täter ….................................................................. 117

5.3.3. Opfer ..................................................................... 119

5.3.4. Ermittler ........................................................................ 120

5.3.5. Frauen - Männer ................................................................ 121

5.3.6. Filmische Präsentation des Serienmördermotivs .................. 122

5.3.7. Zur Funktion des Serienmördermotivs in BIZARRE MORDE .... 130

5.3.8. Resümee ....................................................................... 131

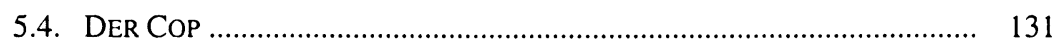

5.4.1. Sequenzprotokoll .......................................................... 132

5.4.2. Ermittler ........................................................................ 134

5.4.3. Opfer ...................................................................... 137

5.4.4. Täter ............................................................................... 138

5.4.5. Frauen - Männer ............................................................. 139

5.4.6. Filmische Präsentation des Serienmördermotivs ................... 141

5.4.7. Zur Funktion des Serienmördermotivs in DER COP ............... 147

5.4.8. Resümee ..................................................................... 148

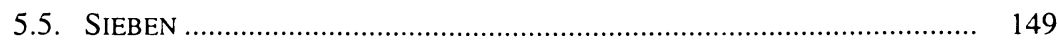

5.5.1. Sequenzprotokoll .......................................................... 150

5.5.2. Ermittler ................................................................ 151 
5.5.3. Opfer

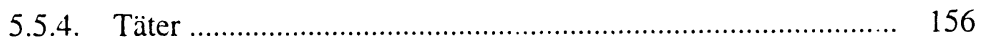

5.5.5. Frauen - Männer ................................................................. 157

5.5.6. Filmische Präsentation des Serienmördermotivs .................... 158

5.5.7. Zur Funktion des Serienmördermotivs in SIEBEN ................... 164

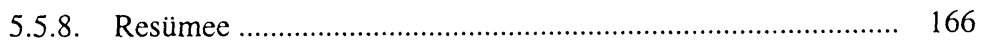

5.6. Täter, Opfer, Ermittler und Gewalt im Serienmördermotiv .................. 168

6. Kulturelle und soziale Bezüge in Verfilmungen des Serienmördermotivs ........ 179

6.1. Der Serienmörderfilm und seine Entsprechung zur Wirklichkeit ........ 179

6.2. Serienmörderkriminalität in der sozialen Wirklichkeit ........................ 184

6.3. Geschlecht, Gender und das Serienmördermotiv ................................ 189

6.4. Die Faszination der Serienmörder .................................................... 199

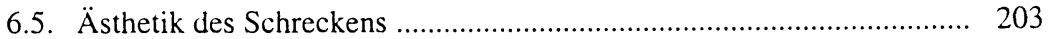

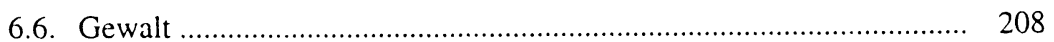

6.7. Gewalt im Serienmörderfilm ....................................................... 211

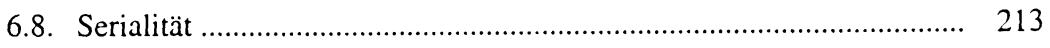

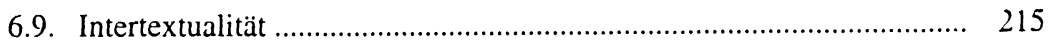

6.10. Die Funktionalisierung des Serienmördermotivs ............................... 217

6.11. Entgrenzung .............................................................................. 220

7. Abschließende Reflexionen über das Serienmördermotiv im Spielfilm ............ 231

7.1. Motivuntersuchungen im filmwissenschaftlichen Kontext ................... 231

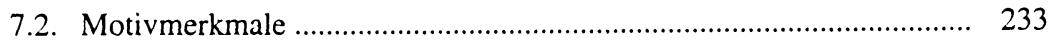

7.3. Übergreifende Bedeutungen des Serienmördermotivs ......................... 237

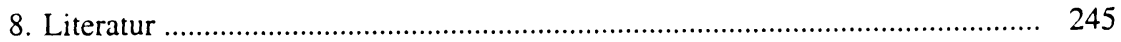

8.1. Bücher und Artikel mit Autorennennung .......................................... 245

8.2. (Zeitschriften-) Beiträge ohne Autorennennung ................................. 266

8.3. Bildquellen ................................................................................ 267

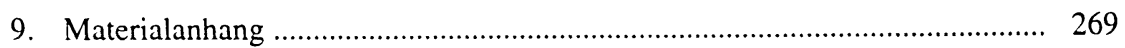

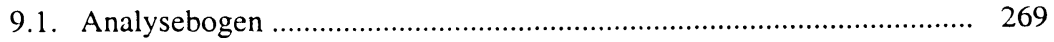

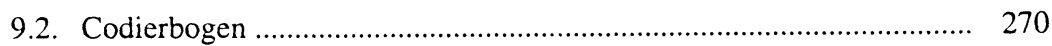

9.3. Ergebnisse der quantitativen Analyse für BIZARRE MORDE, DER COP

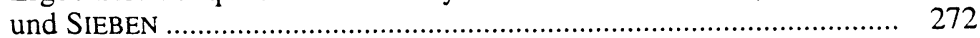

9.4. Weitere Ergebnisse der inhaltsanalytischen Auswertung...................... 273

9.5. Motivinventar zum Serienmördermotiv ............................................. 275

9.6. Serienmörderfilme in alphabetischer Reihenfolge .............................. 278 


\section{Verzeichnis der Abbildungen}

Abbildung 1: Abbildung 2: Abbildung 3: Abbildung 4: Abbildung 5:

Abbildung 6: Abbildung 7: Abbildung 8: Abbildung 9: Abbildung 10: Abbildung 11: Abbildung 12: Abbildung 13:

Abbildung 14: Abbildung 15: Abbildung 16: Abbildung 17: Abbildung 18: Abbildung 19: Abbildung 20: Abbildung 21: Abbildung 22: Abbildung 23: Abbildung 24: Abbildung 25: Abbildung 26: Abbildung 27: Abbildung 28: Abbildung 29: Abbildung 30: Abbildung 31: Abbildung 32: Abbildung 33: Abbildung 34: Abbildung 35: Abbildung 36: Abbildung 37: Abbildung 38: Abbildung 39: Abbildung 40: Abbildung 41: Abbildung 42: Abbildung 43: Abbildung 44 Abbildung 45:

Schatten des Täters, aus: M-EINE STAD' SUCHT EINEN MÖRDER ..

Filmplakat zu Prom Night

Plakat zu ES GESCHAH AM HELLICHTEN TAG ................................. 53

Filmplakat zu SCHATTEN UND NEBEL .................................... 56

Peter Lorre als Kindermörder in M-EINE STADT SUCHT EINEN MÖRDER

Filmstill aus DER GREIFER

Plakat zu EIN MANN WIE DYNAMIT ............................................ $\quad 70$

Filmplakat zu AMERICAN KILLER ............................................ 73

Filmplakat zu NEW YORK RIPPER ........................................... 75

Filmplakat zu Blue Steel .......................................................... 78

Filmplakat zu DER WOLF HETZT DIE MEUTE. ............................... 81

Filmstill aus FRENZY .............................................................. 91

Filmstill aus BECKMANN UND MARKOWSKI: VOM ZWEIFEL DER

GEFÜHLE

Filmplakat zu PSYCHO ................................................................. 99

Filmstill aus DER NEW YORK RIPPER ……................................ 100

Filmstill aus BIZARRE MORDE (Seq. 17) ..................................... 115

Filmstill aus BIZARRE MORDE (Seq. 17) ...................................... 123

Filmstill aus BIZARRE MORDE (Seq. 1) ........................................ 125

Filmstill aus BIZARRE MORDE (Seq. 16) ..................................... 126

Filmstill aus DER COP (Seq. 1) ..................................................... 134

Filmstill aus DER COP (Seq. 13) ................................................ 135

Filmstill aus DER COP (Seq. 11) ............................................. 144

Filmplakat zu DER COP ............................................................. 145

Filmstill aus SEVEN (Seq.2) ................................................... 155

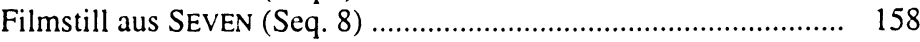

Filmstill aus SEVEN (Seq. 5) ....................................................... 159

Filmstill aus SEVEN (Seq. 8) ................................................. 163

Filmplakat zu CITY COBRA ....................................................... 168

Filmplakat zu BLUTMOND ........................................................ 174

Filmstill aus BLINDFOLD ........................................................... 183

Filmstill aus HalloweEN - DER FluCH DES MICHAEL MYERS .... 183

Filmstill aus DER SERIENKILLER - KLINGE DES TODES .................. 183

Filmstill aus BLACKOUT ......................................................... 183

Filmstill aus THE LODGER ........................................................ 183

Filmstill aus DAS GEHEIMNIS DER SCHWARZEN HANDSCHUHE ..... 189

Filmstill aus DER NEW YORK RIPPER ......................................... 190

Filmstill aus MORD BIZARR .................................................. 190

Zeitungsanzeige zu SCREAM .................................................... 192

Filmstill aus PSYCHO ......................................................... 194

Filmstill aus BLINDFOLD .......................................................... 195

Videocover zu IM AUGENBLICK DER ANGST .................................. 197

Otto Dix: Der Lustmörder ........................................................ 202

TUI-Reklame, aus: Stern 44/1995 ............................................. 213

Filmplakat zu SIEBEN MONDE ................................................. 216 


\section{Verzeichnis der Diagramme}

Diagramm 1: Produktionsjahre der in Deutschland gezeigten

Serienmörderfilme ................................................................... 38

Diagramm 2: Erstaufführungen von Serienmörderfilmen in Deutschland ........... 41

Diagramm 3: Erstausstrahlungsmedien von Serienmörderfilmen in Deutschland 42

Diagramm 4: Genrezugehörigkeit der Serienmörderfilme ............................... 47

Diagramm 5: Protagonisten im Serienmörderfilm ........................................... 54

Diagramm 6: Geschlecht der Serienmörder in \% der Serienmörderfilme ............ $\quad 60$

Diagramm 7: Geschlecht der Opfer ................................................................ 71

Diagramm 8: Handlungsanteil der Opfer .................................................... $\quad 74$

Diagramm 9: Rezeptionsangebote ….......................................................... 83

Diagramm 10: Gewaltdarstellungsintensität ....................................................... 86

Diagramm 11: Historische Entwicklung der Gewaltdarstellungsintensität in \% der untersuchten Filme .............................................................. 88

Diagramm 12: Verwendung mehrerer Spannungselemente pro Spielfilm ........... 96

Diagramm 13: Serienmörderspielfilme und Serienmörderfälle in den USA ......... 181

\section{Verzeichnis der Tabellen}

Tabelle 1: Produktionszahlen von Serienmörderfilmen

Tabelle 2: Anteil der Serienmörderfilme an den in Deutschland erstaufgeführten Spielfilmen

Tabelle 3: Erstaufführungsmedien von Serienmörderfilmen in Deutschland in absoluten Zahlen und als prozentualer Wert

Tabelle 4:

TV-Produktionen zum Thema Serienmörder

Tabelle 5:

Serienmörderfilme und Produktionsländer

Tabelle 6:

Genrezugehörigkeit der Serienmörderfilme

Tabelle 7:

Hauptpersonen in Serienmörderfilmen

Tabelle 8:

Protagonisten in Serienmörderfilmen des Horrorgenres

einschließlich Slasher

Tabelle 9:

Protagonisten in Serienmörderfilmen des Thrillergenres

Tabelle 10:

Tabelle 11:

Protagonisten in Serienmörderfilmen des Krimigenres

Tabelle 12:

Serienmörder nach Tätertypen klassifiziert

Tabelle 13:

Tabelle 14:

Spezielle Tatmotive der Filmserienmörder

Historische Entwicklung der Täterausschaltung ................................ 69

Tabelle 15:

Geschlecht der führend ermittelnden Polizisten

Rolle der Polizei in absoluten und Prozentzahlen ............................ $\quad 79$

Tabelle 16:

Besondere Ermittlercharakteristika

Tabelle 17:

Darstellung von Sex- und von ausgedehnten Gewaltszenen im

Serienmörderfilm

Tabelle 18

Slasherfilme mit Serienmördermotiv

Tabelle 19:

Spannungselemente im Serienmörderfilm

Tabelle 20:

Sequenzgraphik zu BIZARRE MORDE

Tabelle 21:

Tabelle 22:

Tabelle 23:

Zahl der Einstellungen in BIZARRE MORDE pro Minute

Gewaltakte in BIZARRE MORDE 
Tabelle 25: Gewaltakte in DER COP ............................................................... 143

Tabelle 26: Sequenzgraphik zu SIEBEN ..................................................... 150

Tabelle 27: Zahl der Einstellungen in SIEBEN pro Minute ............................... 160

Tabelle 28: Gewaltakte in SIEBEN ................................................................ 162

Tabelle 29: Zeitliches Ausmaß der Gewaltdarstellungen in den drei

Beispielfilmen ..................................................................... 176

Tabelle 30: Serienmörderfälle und Opferzahlen in den USA nach Egger (1990) . 184

Tabelle 31: Frequences of Serial, Mass and all Homicides ............................... 186

Tabelle 32: Morde in den USA ........................................................................ 187

Tabelle 33: Absolute Mordzahlen und Häufigkeitsziffern pro 100.000

Einwohner für die Bundesrepublik Deutschland und die USA ......... 209

Tabelle 34: Absolute Vergewaltigungszahlen und Häufigkeitsziffern pro

100.000 Einwohner für die Bundesrepublik Deutschland und die USA

Tabelle 35: Tötungsarten im Serienmörderfilm ............................................... 273

Tabelle 36: Handlungsorte des Serienmörderfilms ........................................ 273

Tabelle 37: Storyvarianten des Serienmördermotivs ...................................... 274 